\section{Cutaneous and Renal Glomerular Vasculopathy cases in emergency veterinary practices in the UK}

\section{Mariana Abreu ${ }^{1}$, Rodolfo Oliveira Leal ${ }^{2}$, Sarah Ann Ambler ${ }^{3}$}

1 Faculty of Veterinary Medicine, University of Lisbon, Lisbon, Portugal

2 CIISA Centre for Interdisciplinary Research in Animal Health - Faculty of Veterinary Medicine, University of Lisbon Lisbon, Portugal

3 VetsNow, Stoke-on-Trent, United Kingdom

\section{OBJECTIVES}

This study aims to: a) review cases of dogs with suspected cutaneous and renal glomerular vasculopathy (CRGV), evaluating if the cutaneous lesions correlated with the development of acute kidney injury (AKI), b) to assess negative prognostic factors in dogs with suspected CRGV.

\section{METHODS}

A retrospective study including dogs with suspected CRGV presented to 26 first opinion emergency practices in the United Kingdom. Their history, clinical signs, clinicopathological findings, diagnostics, treatment plan, and outcome were reviewed.

\section{RESULTS}

40 dogs were included. 27 presented skin lesions and 13 showed skin lesions and AKI. The most common macroscopic aspects of the skin lesions both in dogs with and without AKI, were superficial abrasions, cutaneous ulcers, inflammation, dermatitis, alopecia, erythema and oedema, principally when located in the limbs and digits. Lesions wider than five centimetres were significantly correlated with the development of AKI ( $p=0.029)$. Dogs with AKI presented with azotaemia anaemia, proteinuria, haematuria, hyposthenuria, hypocalcaemia, thrombocytopenia, neutrophilia, high serum liver enzyme activity, and hyperbilirubinaemia. Five animals were submitted for euthanasia (38.5\%). Azotaemia ( $p=0.001)$, oligoanuria $(p<0.001)$, hypocalcaemia $(p=0.003)$ and hypophosphatemia $(p<0.001)$ were significantly correlated with a worse outcome. Intensive medical therapy is designated to these patients since successful outcomes with full recovery from $\mathrm{AKI}$ have been achieved, as observed in seven dogs $(53.8 \%)$.

\section{STATEMENT (CONCLUSIONS)}

More than contributing to an increased number of reported cases, this study confirms that azotaemia, oligoanuria, hypocalcaemia, and hypophosphatemia are associated with a negative outcome in dogs with suspected CRGV.

\section{Leptospirosis in the East of England}

\section{Jennifer Raj ${ }^{1,2}$, Ruth Campbell ${ }^{1}$, Simon Tappin ${ }^{1}$}

1 Dick White Referrals, Six Mile Bottom, United Kingdom

2 Southfield Veterinary Specialists, Basildon, United Kingdom

\section{OBJECTIVES}

To describe the clinical findings and outcome of dogs diagnosed with leptospirosis in the East of England.

\section{METHODS}

Retrospective review of the medical records of dogs diagnosed with Leptospirosis from February 2008 to July 2019 from a single referral centre. Diagnosis was made on the basis of microscopic agglutination testing (MAT), serology by immunofluorescence, polymerase chain reaction (PCR) on blood, or fluorescence in-situ hybridisation (FISH) on liver biopsy specimens.

\section{RESULTS}

Thirty-eight dogs were included. Clinical signs included inappetence $(76 \%)$, vomiting $(68 \%)$, lethargy (58\%), polydipsia and polyuria (18\%), abdominal pain $(16 \%)$, pyrexia (11\%), weight loss $(8 \%)$, joint pain $(3 \%)$. Clinicopathological findings at presentation included anaemia (42\%), thrombocytopenia (43\%), abnormalities of coagulation times (16\%), increased alanine transaminase (66\%), hyperbilirubinaemia (55\%), azotaemia (68\%), hypoalbuminaemia (49\%), glucosuria (32\%). Disease manifestations included hepatic and renal (47\%), hepatic only (37\%), renal only (16\%); no dogs suffered pulmonary involvement. Seven serovars were detected, the most prevalent was Copenhageni (48\%), followed by Canicola (19\%). All dogs were treated with antibiotics; other treatments included anti-emetics (79\%), analgesia (53\%) and gastroprotective medication (32\%). Seventy-one percent survived to discharge. Dogs in the non-surviving group had a higher creatinine at presentation compared to survivors $(p=0.02)$.

\section{STATEMENT (CONCLUSIONS)}

Copenhageni is the most prevalent serovar in these referral cases from the East of England which supports quadrivalent vaccine use in this location. A hepatic only form appears to be common in this region so should be considered a differential diagnosis in dogs presenting with signs of hepatic disease. 